\title{
An Application of Mobile Crowdsensing Techniques: Multiple Physiological Parameters Detection System
}

\author{
Liu-bin $\mathrm{LI}^{1^{*}}$, Liu WANG ${ }^{2}$, Song MA ${ }^{3}$, Kai $\mathrm{LI}^{4}$, Guan WANG ${ }^{5}$ and \\ Yu-hua CHENG ${ }^{6}$ \\ ${ }^{1}$ Shanghai Research Institute of Microelectronics(SHRIME), Peking University, \\ ShangHai, China \\ ${ }^{2}$ Shanghai Research Institute of Microelectronics(SHRIME), Peking University, \\ ShangHai, China \\ ${ }^{3}$ Shanghai Research Institute of Microelectronics(SHRIME), Peking University, \\ ShangHai, China \\ ${ }^{4}$ Shanghai Research Institute of Microelectronics(SHRIME), Peking University, \\ ShangHai, China \\ ${ }^{5}$ Shanghai Research Institute of Microelectronics(SHRIME), Peking University, \\ ShangHai, China \\ ${ }^{6}$ Shanghai Research Institute of Microelectronics(SHRIME), Peking University, \\ ShangHai, China, and School of Electronics Engineering and Computer Science \\ (EECS), Peking University, Beijing, China. \\ ${ }^{1,2,3,4,5,6}$ NO.608, Shengxia Road, Pudong New Area \\ *liiubin@shrime-pku.org.cn
}

Keywords: Physiological Parameters Detection, loT, Mobile Crowdsensing, Health care .

\begin{abstract}
Mobile crowdsensing serves as a critical building block for the emerging Internet of Things(IoT). Smartphones is a typical example of mobile sensing device. In this paper, we will introduce an application based on mobile crowdsensing[1], which is multiple physiological parameters detection system. Elderly and pregnant are both of vulnerable groups in China, and the number of these people is huge. The healthy status of these people is our main concern. With the multiple physiological parameters, we can forecast some kind of disease. Hypertension,for example. Firstly, the PPG(photoplethysmography) signal will be got by the MCU. Secondly, the raw data will be send to the mobile app through bluetooth. Finally, the result will be send to the cloud. If any abnormal data occurs, targeted services will be provided for the users.
\end{abstract}

\section{Introduction}

Technology related to smart phone is developing fast in every field. The developing of health care is outstanding. However, the status of the elderly is getting worse for the number of the elderly is growing fast. Figure 1 [2] shows the trend of the developing of the elderly in China. We can learn that China is an elderly country with the elderly percentage of $15 \%$ now, which is far beyond the standard $7 \%$. Besides, this percentage will get worse to $33 \%$ till 2050 . That's to say one of three is an elder. 


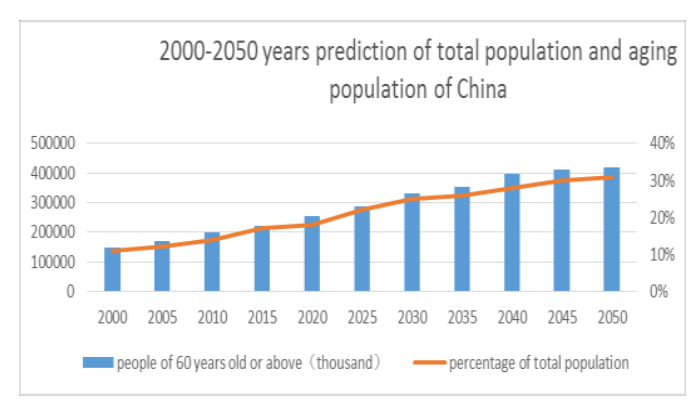

Figure 1 Forecast of the Elderly In China

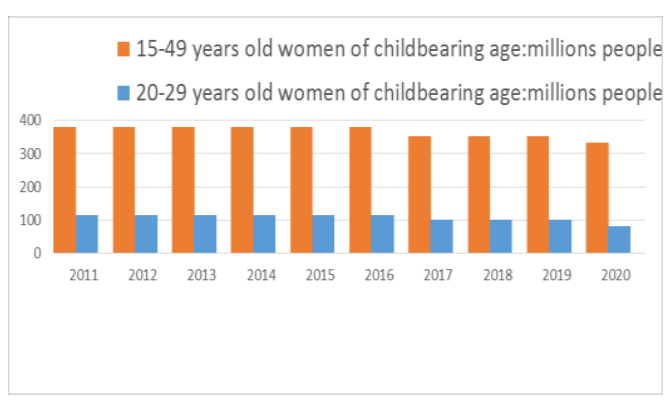

Figure 2 Forecast of Women Fit Pregnant

Figure 2[2] shows the trend of the women fit pregnant. Though the total is decreasing, the number is still very large. These two kind of people are the main target of our system[3].

\section{Realization of the Multiple Physiological Parameters Detection System}

\section{Principle and Method of Calculation}

The physiological parameters includes blood pressure, heart beat, blood oxygen, respiratory rate and temperature. Temperature is measured by a temperature sensor, other parameters are measured using the PPG(photoplethysmography) signal.

Blood pressure:

Figure 3 shows the principle of calculating the systolic blood pressure. The upper one is the PPG pulse wave signal, the lower one is the accelerated pulse wave. When the heart shrinks, the blood is pumped to the vessels from the heart, so there will be an slop upward in the pulse wave, that's to say the vessel wall will sensing the pressure at point A. While Venous blood flow is larger, when the blood coming from the heart flow into the vein, there will be a backflow to the heart. The backflow point is point $C$. The time between this two point is called PWTT(Pulse Wave Transport Time) ${ }^{[4,5]}$. When the systolic pressure is high, the PWTT will be smaller. The diastolic pressure is an empirical formula, which is concluded from a large amount of clinical trials.
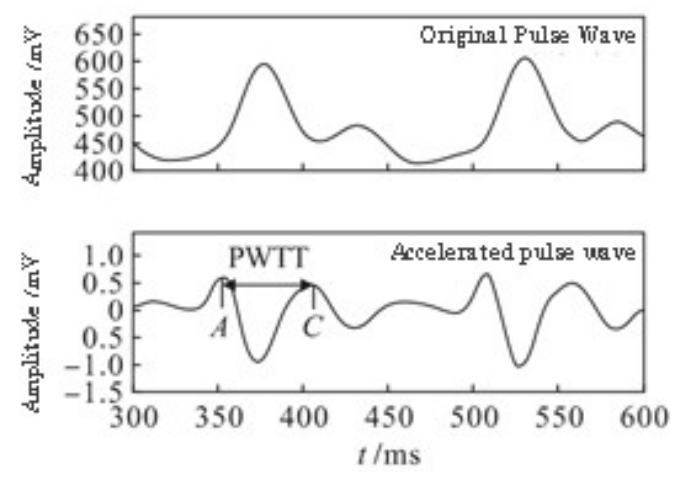

Figure 3 Pulse Wave and Accelerate Pulse Wave

Blood oxygen:

When a beam of monochromatic light pass through homogeneous solution of a certain substance, the light is partly absorbed. Lambert-beer's law ${ }^{[6]}$ described the elationship of different factors before and after light pass through the solution. With the heart is contracting and relaxing, blood pumps and returns, and then the wall of the capillary will occur periodically in the expansion and contraction. From the analysis of Lambert-beer's law, we can learn that the intensity of the light passing through is changed. Apart from this part, some other parts human body such as Skin, muscle, bone, 
venous blood and arterial blood. However, the light intensity of passing through these organization is supposed to be stable. So the changing part of the transmitted light is regarded as the $\mathrm{AC}$ component and the stable part is regarded as the $\mathrm{DC}$ component.

$$
S p O_{2}=\frac{\varepsilon_{2} \frac{D^{\prime}}{D}-\varepsilon_{1}}{\left(\varepsilon_{2}-\varepsilon_{1}\right) \frac{D^{\prime}}{D}-\left(\varepsilon_{2}^{\prime}-\varepsilon_{1}^{\prime}\right)}
$$

Where $D / D^{\prime}=\left(A C_{\lambda_{1}} / D C_{\lambda_{1}}\right) /\left(A C_{\lambda_{2}} / D C_{\lambda_{2}}\right)$ This ratio is usually referred to as the oxygen saturation coefficient. Oxygen saturation can be obtained further.

\section{Hardware Design of the System}

This section describes the signal architecture of the whole system. Figure 4 is the whole data path of the design. Firstly, the data got by MCU is transported to the APP on the mobile through the bluetooth. Secondly, the algorithm will be executed to get the result. Finally, the result will be send to the cloud to form the personal database, which will be used to get personal care.

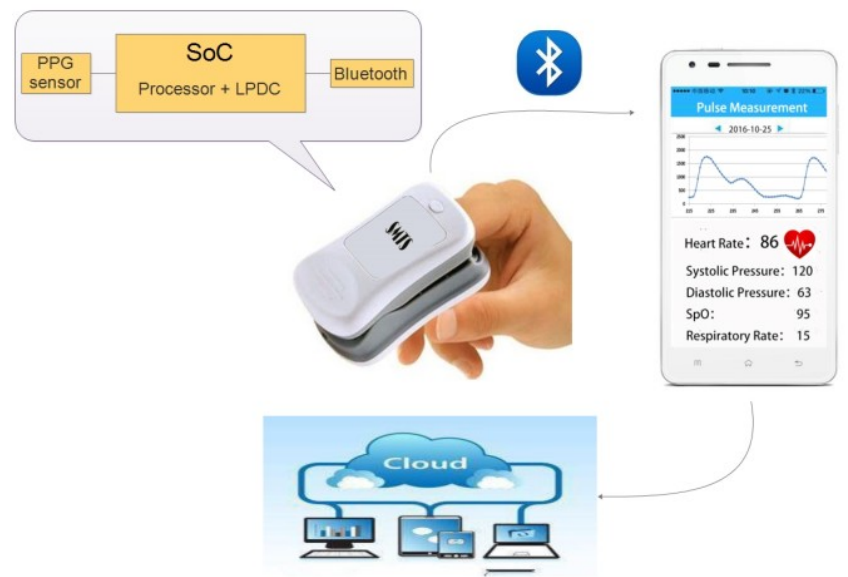

Figure 4 Datapath of Multiple Physiological Parameters Detection System

As mentioned above, blood oxygen detect needs two strobe of lights, that's both the DC and AC voltage will be sampled. To simply the and lower the cost or the circuits, the red and infrared light is working time-sharing.

\section{Security and Reliability[7,8]}

In our society, your private information will be exposed once you register a website or something else. So some people is unwilling to use a product if the personal information is not well protected, not even talking about share personal data to others. Besides, if the result of our system is not taken seriously, some kind of disease will not be found timely. Considering these, we choose to cooperate with hospitals, the results will be seen by the doctor as well if the result is abnormal. What's more, to ensure the reliability we added the $\mathrm{CRC}$ check algorithm to ensure the data transported is absolutely the data we want to send.

\section{Result and Discussion}

As mentioned above, the data sampled by the MCU is send to the smartphone. Figure 5 shows the data processing procedure. 


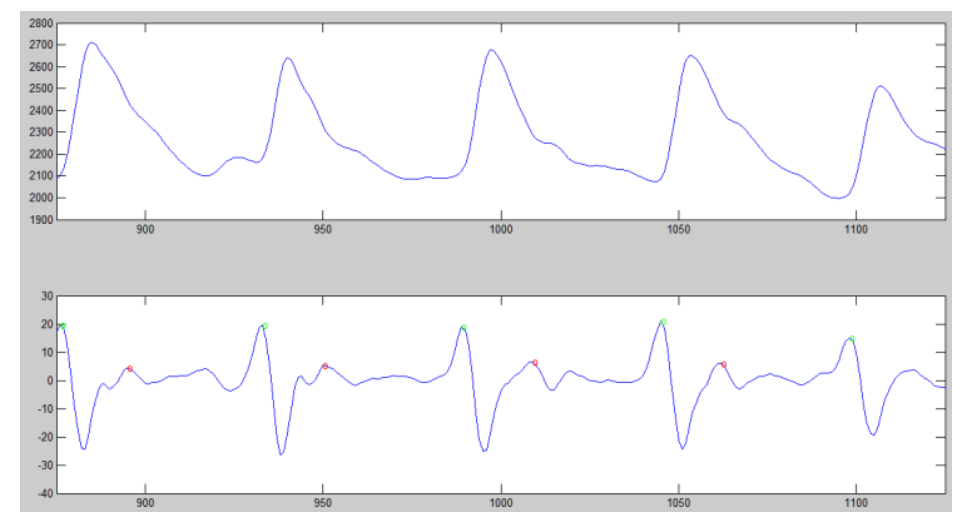

Figure 5 The Pulse Wave and The Derived Accelerate Pulse Wave

The PWTT of the above signal is 20.976, The SBP(systolic blood pressure) computed is 112.11. The synchronous blood pressure tested by the arm blood test equipment is 115 . For a large amount of data, the SBP error compared with the standard blood pressure is within $3 \mathrm{mmHg}$. Though we can get the SBP precisely, there is still some problem with the DBP. That is the error of DBP is up to $10 \mathrm{mmHg}$. However, the SBP means more than DBP. Besides, other parameters detected by this system is reliable.

\section{Conclusion and Future Work}

Most of the advanced technological futures are for the young people. But as we early discussed, the percentage of the elderly people is increasing day by day. This paper developed a system for multiple physiological parameters detecting. With the sensor and hardware circuits, the smartphone can get the personal physiological parameters. With the smartphone, all the data will be transfer to the cloud. In the future, we will complete the diastolic blood pressure and we will take action movement as an added function[9,10].

\section{Acknowledgements}

This research was supported by the Special Development Fund of Shanghai Zhangjiang National Innovation Demonstration Zone(201411-PD-JQ-B108-004), 2015 innovation fund of Pudong New District science and technology development Program, 863 National High Technology Research and Development Program of China (2013AA011202), and National 02 Key Special Program (2012ZX02503-005).

\section{References}

[1] C Luo, T Y Li, X L. Quan, et al. A new algorithm of blood pressure measurement based on oscillometric method [J]. Automation Congress, 2008, 1-4.

[2] China Industrial Information Network, http://www.cnii.com.cn/

[3] Jer-Vui Lee, Yea-Dat Chuah and Kenny T.H. Chieng, "Smart Elderly Home Monitoring System with an Android Phone", International Journal of Smart Home, Vol. 7, No. 3, pp. 17-30, May- 2013.

[4] Y. Chen, C Wen, G C Tao, et al. Continuous and noninvasive blood pressure measurement: anovel modeling methodology of the relationship between blood 
pressure and pulse wavevelocity [J]. Annals of Biomedical Engineering, 2009,37(11): 2222-2233.

[5] C Luo, T Y Li, X L. Quan, et al. A new algorithm of blood pressure measurement based on oscillometric method [J]. Automation Congress, 2008, 1-4.

[6] R.C. Gupta, S.S. Ahluwalia, S.S. Randhawa. Design and Development of Pulse Oximeter. Proceedings RCIEEE-EMBS \&14th BMESI, 1995:pp.13-16

[7] M. Arapinis, L. Mancini, E. Ritter, and M. Ryan, "Privacy through pseudonymity in mobile telephony systems," in NDSS, 2014.

[8] L. Sweeney, "k-anonymity: A model for protecting privacy," International Journal of Uncertainty, Fuzziness, and Knowledge-based Systems, vol. 10, no. 5, pp. 557-570, October 2002.

[9] Jessica Cocco, "Smart Home Technology for the Elderly and the Need for Regulation", Journal of Environmental and Public Health Law, Volume 6-Isssu 1, pp. 85-108, Winter 2011.

[10]S. Saroiu and A. Wolman, "Enabling new mobile applications with location proofs," in Proc. of HotMobile, 2009, pp. 1-6. 